[0212-7199 (2004) 21: 9; pp 425-432] ANALES DE MEDICINA INTERNA Copyright @ 2004 ARAN EDICIONES, S.L.

AN. MED. INTERNA (Madrid) Vol. 21, N. ${ }^{\circ}$, pp. 425-432, 2004

\section{Bacteriemia por anaerobios: características clinicoepidemiológicas de las bacteriemias por anaerobios en dos periodos con una diferencia de 10 años}

\author{
J. M. RUIZ-GIARDÍN, A. NOGUERADO ASENSIO' \\ Servicio de Medicina Interna. Hospital Universitario de La Princesa. \\ ${ }^{1}$ Hospital Universitario Gregorio Marañón-Cantoblanco. Madrid
}

ANAEROBIC BACTEREMIAS: CLINICAL AND EPIDEMIOLOGICAL CHARACTERISTICS OF ANAEROBIC BACTEREMIAS IN TEN YEARS

\section{RESUMEN}

Fundamento: La incidencia de bacteriemia por anaerobios parece que está disminuyendo en los últimos años, y pese a la elevada mortalidad de las mismas existen estudios que plantean la supresión de medios de crecimiento para anaerobios basándose en la predictibilidad de dichas bacteriemias. El objetivo del estudio es analizar la incidencia, y características clínicas de las bacteriemias por anaerobios en dos periodos con 10 años de diferencia, analizando si el conocimiento de dichos microorganismos modificó las decisiones terapéuticas tomadas empíricamente antes del conocimiento de los mismos.

Métodos: Estudio prospectivo y anterógrado de las bacteriemias por anaerobios durante 1985-86 y 1996-97 en un hospital universitario, analizando su incidencia, características clínicas, analíticas y su evolución a curación o muerte. Para el análisis estadístico se realizó test de chi cuadrado o test exacto de Fisher, considerándose estadísticamente significativas $\mathrm{p}<0,05$, comentándose las variables con $\mathrm{p}<0,10$.

Resultados: La incidencia de bacteriemia por anaerobios (sobre el total de las bacteriemias diagnosticadas) fue superior en 1996-97 con 24 casos $(5,08 \%)$, que durante $1985-86$ con 22 casos $(4,24 \%)$. Las variables con diferencias estadísticamente significativas con $\mathrm{p}<0,10$ fueron: adquisición intrahospitalaria $(\mathrm{p}<0,10)$; manipulaciones genitourinarias ( $\mathrm{p}=0,02)$; manipulaciones vasculares $(\mathrm{p}$ de 0,06$)$ y la presencia de hipotensión $(\mathrm{p}=0,034)$ más frecuentes durante el primer periodo que en el segundo. No se observa diferencia estadísticamente significativa en cuanto a la evolución a curación aunque el porcentaje de estas fue superior durante el segundo periodo $(62,5 \%)$ que durante el primero $(54,6 \%)$, siendo el número de tratamientos empíricos adecuados muy elevado en ambos (77 y $87 \%$ respectivamente). En ambos periodos el germen más frecuentemente aislado fue Bacteroides fragilis, y el foco de origen más frecuente fue el abdominal.

Conclusión: Aunque la incidencia de bacteriemias por anaerobios es baja, esta no ha disminuído en 10 años. Las bacteriemias por anaerobios tienen una elevada mortalidad pese a que la mayor parte de ellas reciben tratamiento empírico adecuado. Todo ello podría plantear la posibilidad de suprimir los medios de cultivo para anaerobios, o solicitarlos en casos clínicamente sospechosos.

PALABRAS CLAVE: Bacteriemia. Anaerobios. Incidencia. Hemocultivos.

\section{ABSTRACT}

Background: The anaerobic bacteremia incidence is decreasing during the last years, and although it carries a high mortality rate there are studies that ask about the posibility of avoid anaerobic blood cultures thinking about the clinical prediction capacity of them. The objective of this study is the analisis of incidence and clinical characteristics of anaerobic bacteremias in two periods with 10 years of difference and empiric antibiotic treatment received, and if it was changed when microbiological results were received.

Methods: Prospective study of anaerobic bacteremias during 198586 and 1996-97, at university hospital analizing clinical characteristics, incidence, analisis, evolution and empiric antibiotic treatment received. The statistical analysis was performed with the chi square test or exact Fisher test with satistical significance with $p<0.05$, talking about variables with $p<0.10$.

Results: The incidence of anaerobic bacteremias was higher in 1996-97 with 24 cases (5.08\%), that during 1985-86 with 22 cases (4.24\%).

The variables with statistical significative differences with $p<0.10$ were: intrahospitalary adquisition $(p<0.10)$; genitourinary and vascular manipulation $(p=0.02$ and $p=0.06)$, and hipotension $(p=0.034)$ more frequent during the first period than during the second one. There isn't statistical difference in evolution to cure although the percentage was higher during the second period (62.5\%), that during the first one (54.6\%), being the empiric treatment very high in both periods. Bacteroides fragilis was the most frequent microrganism and abdominal origin the most frequent one in both periods.

Conclusion: Although there is a low anaerobic bacteremia incidence this one hasn't diminished in ten years. Anaerobic bacteremias have a high mortality index although the most part of empirical antibiotic treatments are correct. It could ask about the posibility of avoid anaerobic blood cultures or ask about them only in clinic suspicion of them.

KEY WORDS: Bacteremia. Anaerobes. Incidence. Blood cultures.

Ruiz-Giardín JM, Noguerado Asensio A. Bacteriemia por anaerobios: características clínicoepidemiológicas de las bacteriemias por anaerobios en dos periodos con una diferencia de 10 años. An Med Interna (Madrid) 2004; 21: 425-432. 


\section{INTRODUCCIÓN}

Las bacterias anaerobias forman parte de la flora de distintos órganos y sistemas del cuerpo humano, tales como aparato digestivo, aparato genital femenino, piel o vías respiratorias superiores. El reconocimiento de las bacteriemias por anaerobios es importante ya que presentan una elevada mortalidad cuando no son tratadas adecuadamente (1-3), aunque existe algún estudio en el que no existe diferencia en el curso clínico de los pacientes que recibían tratamiento adecuado o inadecuado (4).

Las bacteriemias por anaerobios constituyen entre el 1,8 y el $25 \%$ de los aislamientos en hemocultivos (clínicamente significativos) (2,5-6); (7\%) (4), (2\%) $(3,7)$.

El germen más frecuentemente aislado en las bacteriemias por anaerobios es el Bacteroides fragilis $(4,8)$ que en la mayor parte de las series supone más del $50 \%$ de los aislamientos $(2,9,10)$.

Existen estudios que afirman que las bacteriemias por anaerobios están disminuyendo en los últimos años, mientras que las fungemias están aumentando (11,12). El motivo de tales cambios están poco claros, pero podrían estar relacionados con un diagnóstico y tratamiento precoz de las infecciones localizadas por anaerobios, el uso de profilaxis adecuadas preoperatorias a la cirugía intestinal, y al uso de antimicrobianos de amplio espectro que incluyen agentes con actividad frente a anaerobios.

El objetivo del estudio es:

Comparar la incidencia de las bacteriemias por anaerobios en 10 años de evolución, comparando el periodo 1985-1986, frente al periodo 1996-97.

Comparar las características clínicas, analíticas y evolución de dichas bacteriemias en ambos periodos.

Valorar si la realización de hemocultivos en medios de crecimiento para anaerobios, modifica el tratamiento médico pautado empíricamente antes del conocimiento del microrganismo anaerobio, para valorar la posible utilidad o no de estos medios de cultivo en la práctica clínica habitual.

\section{MÉTODO}

Ámbito de estudio: Hospital de La Princesa, universitario, con 500 camas y cobertura para 450.000 habitantes.

Durante los periodos, 1 de enero de 1985 y el 31 de diciembre de 1986, y el 16 de mayo de 1996 al 14 de mayo de 1997 se siguieron de forma prospectiva a todos los pacientes que presentaron hemocultivo clínicamente positivo, descartándose los contaminantes tras la valoración del paciente, siguiendo la evolución de los pacientes hasta la curación del episodio o fallecimiento. Para tal proceso, y de acuerdo con el servicio de microbiología, diariamente se valoraron a todos los pacientes que durante los periodos de estudio presentaron hemocultivos positivos, y se siguieron a los mismos hasta su curación o muerte. Durante dicho proceso se recogieron las variables que se definen posteriormente.

Diseño: Estudio prospectivo, concurrente y anterógrado.

Se ha utilizado la misma metodología microbiológica y definiciones clínicas en los dos periodos de estudio. Todos los casos fueron discutidos por el grupo de trabajo. Los datos se recogen en el mismo protocolo en ambos periodos de estudio para su posterior introducción y procesamiento con el paquete estadístico SPSS-PC-V6.04.
PERIODO DE ESTUDIO 1985-1986

Microbiología: La técnica de realización de los hemocultivos se divide en 2 fases: extracción y procesamiento, realizados según protocolo y técnicas habituales $(3,12)$. La extracción se realizó por los ayudantes técnicos sanitarios (ATS) de cada servicio cuando los pacientes tenían fiebre o presentaban escalofríos, tiritona o algún otro síntoma sospechoso de sepsis. Previa esterilización con alcohol yodado se practicó venopunción directa con la extracción de $10 \mathrm{ml}$ de sangre cada 20 minutos y en lugares diferentes, oscilando entre 1 y 3 extracciones dependiendo de las condiciones del paciente; en algunos pacientes hematológicos sometidos a quimioterapia de inducción y en los transplantados de médula ósea, la extracción se realizó a través de catéteres tunelizados. Para su procesamiento, cada muestra se introdujo en dos frascos (set) conteniendo $100 \mathrm{ml}$ de infusión cerebro-corazón (BHI) con $10 \%$ de CO2 y vacío parcial para cultivo de aerobios y $100 \mathrm{ml}$ de medio de Schaedler pre-reducido y suplementado con atmósfera anaerobia y vacío parcial para aislamiento de anaerobios. El caldo de $\mathrm{BHI}$ fue ventilado y ambos frascos se incubaron a $37^{\circ} \mathrm{C}$ después de su extracción. Durante las primeras 12 a 24 horas se realizó tinción con naranja de acridina a partir de frasco aerobio. Los frascos negativos fueron incubados a $37^{\circ} \mathrm{C}$ durante 10 días y revisados diariamente para detectar signos macroscópicos de crecimiento. Si eran negativos a los 10 días se realizó un pase ciego a agar chocolate. Los hemocultivos positivos por la tinción de naranja de acridina o por presentar signos de crecimiento, se procesaron con una tinción de Gram y subcultivo en agar sangre (incubado a $37{ }^{\circ} \mathrm{C}$ durante 18-48 horas en atmósfera aerobia), agar chocolate (incubado a $37{ }^{\circ} \mathrm{C}$ durante $18-48$ horas en atmósfera con $10 \%$ de $\mathrm{CO} 2$ ) y medio de Wilkins-Chalgren (incubado a $37{ }^{\circ} \mathrm{C}$ durante 48 horas en atmósfera anaerobia), así como la determinación de sensibilidad por técnicas de difusión a partir del caldo del hemocultivo. En los casos en los que clínicamente existía sospecha de brucelosis o fungemia, los frascos fueron incubados durante 30 días y cada 7 se realizó un subcultivo ciego en agar chocolate. Los microorganismos crecidos se identificaron mediante las técnicas habituales.

\section{Definiciones}

1. Episodio de BFP verdadera y contaminacion: se consideró BFP verdadera, en base al número de set positivos, los datos clínicos acompañantes, las propias características de los microorganismos y si estos aparecían en otros focos. Cuando no se cumplieron parcial o totalmente estos requisitos se consideró contaminante. En general se consideró el mismo episodio de BFP verdadera cuando se aislaron los mismos microorganismos durante los primeros 7 días. Ocasionalmente, periodos más largos fueron considerados si la situación clínica así lo indicaba.

2. Edad: los pacientes fueron distribuidos en 3 grupos: menores 40 años, 40 a 60 años y mayores de 60 años.

3. Adquisición: se consideró BFP intrahospitalaria cuando el episodio se desarrolló después de 48 horas de estancia en el hospital o si estuvo claramente relacionado con algún procedimiento diagnóstico o terapéutico que se practicara después del ingreso.

4. Servicio: de acuerdo con las características del hospital 
se agruparon en 4 apartados: a) servicios médicos (Aparato Digestivo, Neumología, Neurología, Cardiología, Medicina Interna, Reumatología, Nefrología y Diálisis, Oncología, Dermatología y Enfermedades Infecciosas); b) servicios quirúrgicos (Cirugía General y Digestivo, Cirugía de Tórax, Urología, Traumatología, Otorrinolaringología, Cirugía Cardiovascular y Neurocirugía); c) Hematología incluido transplante de medula ósea; y d) Unidad Cuidados Intensivos.

5. Enfermedad de base: la enfermedad de base se consideró de manera individual siguiendo a Weinstein y cols. (4). Se consideraron: enfermedades hematológicas diagnosticadas por biopsia de médula ósea o ganglionar; tumores sólidos confirmados por biopsia o por evidencia clínica; cirrosis hepática confirmada por datos clínicos y/o anátomo-patológicos; diabetes mellitus establecida previamente o hiperglucemia por encima de $250 \mathrm{mg} \%$; insuficiencia renal considerada si los valores de creatinina sobrepasaron los $2 \mathrm{mg} \%$. Infección VIH con linfocitos CD4 < 500 .

6. Factores predisponentes: se incluyen en este apartado el tratamiento previo con antibióticos a dosis terapéuticas durante los 7 días previos (incluidos los utilizados como profilaxis en pacientes neutropénicos), el uso de citostáticos (incluidos supresores como Ciclosporina A e Inmunoglobulina antitimocítica), radioterapia, y el uso de esteroides a dosis iguales o superiores a $20 \mathrm{mgr} /$ día de prednisona o sus equivalentes durante los 7 días previos. Se consideró neutropenia, cuando la cifra de neutrófilos estaba por debajo de $1.000 / \mathrm{mm}^{3}$.

7. Manipulaciones: se consideró a aquellas maniobras diagnósticas o terapéuticas realizadas durante los 7 días previos al desarrollo de la bacteriemia. Se incluyen manipulaciones digestivas (endoscopia alta y baja, laparoscopia con o sin biopsia), urológicas (sonda vesical, cistografía, cistoscopia con o sin biopsia), respiratorias (intubación, endoscopia con o sin biopsia), vasculares (catéteres intravenosos, marcapasos, fístulas arterio-venosas) y cirugía.

8. Foco de origen: la determinación del foco de origen se basó en hallazgos clínicos y/o microbiológicos. Cuando no se encontró ninguna localización o si los datos fueron confusos se determinó como origen desconocido. La endocarditis se consideró como foco de origen en la mayoría de las ocasiones al no poderse determinar con certeza el foco de bacteriemia inicial. El resto de localizaciones fueron catéteres intravenosos, abdomen, aparato respiratorio, vía biliar, tracto nefrourológico, osteoarticular piel y heridas quirúrgicas.

9. Datos clinicos y de laboratorio: se consideró hipotensión o shock cuando la cifra de tensión sistólica estaba por debajo de $90 \mathrm{mmHg}$ en pacientes normotensos o una caída superior a $70 \mathrm{mmHg}$ sistólica en pacientes hipertensos. Trombocitopenia se definió si la cifra de plaquetas fue inferior a $100.000 / \mathrm{mm}^{3}$. Coagulación intravascular diseminada (CID) se estableció por datos clínicos y/o de laboratorio (disminución del tiempo de protrombina, aumento de tiempo de cefalina $\mathrm{y}$ trombopenia) no explicable por otros procesos. Leucocitosis: Número de leucocitos mayor a 10.000/ml.

10. Tratamiento: se define como tratamiento empírico al realizado después de la extracción de los hemocultivos y hasta la llegada del antibiograma: a) no valorable: cuando el paciente estaba sometido a tratamiento antibiótico previo por otro proceso infeccioso o la muerte se produjo antes de poder instaurarse el tratamiento; b) correcto: episodios donde sólo el tratamiento quirúrgico o la retirada de catéteres, fueron suficiente sin tener que añadir antibióticos; utilización de al menos un antibiótico (posteriormente sensible "in vitro") a dosis y ruta de administración adecuadas y tiempo mínimo de 5 días; y c) tratamiento incorrecto incluyó la no utilización de antibióticos siendo necesaria por la gravedad del proceso; la utilización de antibióticos frente a los cuales el germen presentaba resistencia, dosis insuficientes, duración menor de 5 días y no realización de tratamiento quirúrgico cuando este estaba indicado.

11. Evolución: la curación se estableció mediante datos clínicos y/o microbiológicos después del tratamiento. La muerte del paciente fue valorada en el contexto de su relación directa con la BFP.

\section{PERIODO DE ESTUDIO 1996-1997}

Microbiología: La técnica de realización de los hemocultivos es similar a la fase previa. Sin embargo, el procesamiento actual ha cambiado y cada muestra de $10 \mathrm{ml}$ de sangre se divide en dos de $5 \mathrm{ml}$ y se introduce en dos frascos de $50 \mathrm{ml}$ cada uno para aerobios y anaerobios. Se utiliza el sistema automatizado Bactec NR 730 y se valora como positivo en la lectura automática el valor de 35 (punto de corte). Posteriormente a los frascos positivos se les realiza tinción de Gram siendo el procesamiento posterior similar al descrito en el periodo anterior.

\section{MÉTODO ESTADÍSTICO}

En primer lugar se realizó un estudio de incidencia de bacteriemia por anaerobios en ambos periodos, calculándolo con el numero de bacteriemias por anaerobios en cada uno de los periodos, dividido por el número de bacteriemias verdaderas positivas que aparecieron en cada uno de dichos periodos.

En segundo lugar se hizo un estudio descriptivo de todas las variables estudiadas en las bacteriemias por anaerobios en ambos periodos, analizando mediante estudio chi cuadrado o test exacto de Fisher la existencia de diferencias estadísticamente significativas. La finalidad de esta segunda parte es establecer diferencias descriptivas en las características de las bacteriemias por anaerobios en ambos periodos.

En tercer lugar se analizaron los tratamientos empíricos realizados tras las extracciones de hemocultivos, con intención de conocer si el crecimiento de un microorganismo en el medio de cultivo supuso modificación en la decisión terapeútica previamente tomada. Se calcularon las proporciones de tratamientos empíricos adecuados e inadecuados, así como su relación con evolución a muerte en ambos grupos (tratamientos empíricos adecuados e inadecuados), y en ambos periodos, estudiando igualmente si la diferencia entre proporciones presenta diferencia estadísticamente significativa, mediante el test de Chi cuadrado o test exacto de Fisher. En todos los caso se consideró una p estadísticamente significativa si $\mathrm{p}<0,05$, aunque se mencionan las variables con $\mathrm{p}<0,10$.

Todos los datos fueron procesados utilizando el paquete estadístico SPSS. 


\section{RESULTADOS}

AÑ 1985-86 (TABLA I)

Se obtuvieron un total 11.958 hemocultivos, de los que fueron positivos $1.333(11,1 \%)$, y verdaderos positivos 518 $(38,4 \%)$. De estos, 22 (4,24\%) fueron bacteriemias por anae-

\section{TABLA I}

ANÁLISIS DESCRIPTIVO DE LAS VARIABLES ESTUDIADAS EN LOS PERIODOS 1985-86 Y 1996-97

\begin{tabular}{|c|c|c|c|}
\hline Variable & $1985-86$ & $1996-97$ & $p$ \\
\hline$N^{\circ}$ de hemocultivos & 11.958 & 4.958 & \\
\hline $\begin{array}{l}N^{0} \text { de hemocultivos verdaderos } \\
\text { positivos }\end{array}$ & 518 & 472 & \\
\hline $\begin{array}{l}N^{\circ} \text { de bacteriemias por } \\
\text { anaerobios }\end{array}$ & $22(4,24 \%)$ & $24(5,08 \%)$ & \\
\hline Evolución a muerte & $10(45,4 \%)$ & $9(37,5 \%)$ & 0,58 \\
\hline Edad (años) & & & \\
\hline $\begin{array}{l}<40 \\
\quad 40-60 \\
\quad>60\end{array}$ & $\begin{array}{c}2(9 \%) \\
6(27,2 \%) \\
14(63,6 \%)\end{array}$ & $\begin{array}{l}3(12,2 \%) \\
3(12,2 \%) \\
18(75 \%)\end{array}$ & 0,44 \\
\hline $\begin{array}{l}\text { Sexo } \\
\text { Masculino } \\
\text { Femenino }\end{array}$ & $\begin{array}{l}10(45,4 \%) \\
12(54,6 \%)\end{array}$ & $\begin{array}{l}13(54,2 \%) \\
11(45,8 \%)\end{array}$ & 0,55 \\
\hline $\begin{array}{l}\text { Servicio } \\
\text { Médico } \\
\text { Quirúrgico } \\
\text { UCl } \\
\text { Hematología }\end{array}$ & $\begin{array}{c}15(68,2 \%) \\
3(13,6 \%) \\
3(13,6 \%) \\
1(4,6 \%)\end{array}$ & $\begin{array}{c}13(54,2 \%) \\
9(37,5 \%) \\
2(8,3 \%)\end{array}$ & 0,23 \\
\hline $\begin{array}{l}\text { Adquisición } \\
\text { Extra } \\
\text { Intra }\end{array}$ & $\begin{array}{c}8(36,4 \%) \\
14(83,6 \%)\end{array}$ & $\begin{array}{l}15(62,5 \%) \\
9(37,5 \%)\end{array}$ & 0,07 \\
\hline $\begin{array}{l}\text { Enfermedad de base: } \\
\text { Ninguna } \\
\text { Una o más }\end{array}$ & $\begin{array}{l}12(54,5 \%) \\
10(45,5 \%)\end{array}$ & $\begin{array}{l}12(50 \%) \\
12(50 \%)\end{array}$ & 0,69 \\
\hline $\begin{array}{l}\text { Esteroides } \\
\text { no/sí }\end{array}$ & $\begin{array}{c}21(95,4 \%) \\
1(4,6 \%)\end{array}$ & $\begin{array}{c}23(95,8 \%) \\
1(4,2 \%)\end{array}$ & 0,95 \\
\hline $\begin{array}{l}\text { Antibióticos previos } \\
\text { no/sí }\end{array}$ & $\begin{array}{l}15(68,2 \%) \\
7(31,8 \%)\end{array}$ & $\begin{array}{l}18(75 \%) \\
6(25 \%)\end{array}$ & 0,61 \\
\hline $\begin{array}{l}\text { Inmunosupresores, } \\
\text { no/sí }\end{array}$ & $\begin{array}{c}21(95,4 \%) \\
1(4,6 \%)\end{array}$ & $\begin{array}{c}23(95,8 \%) \\
1(4,2 \%)\end{array}$ & 0,95 \\
\hline $\begin{array}{l}\text { Neutropenia } \\
\text { no/sí }\end{array}$ & $22(100 \%)$ & $\begin{array}{c}23(95,8 \%) \\
1(4,2 \%)\end{array}$ & 1 \\
\hline $\begin{array}{l}\text { Manipulación respiratoria } \\
\text { no/sí }\end{array}$ & $\begin{array}{c}21(95,4 \%) \\
1(4,6 \%)\end{array}$ & $\begin{array}{c}23(95,8 \%) \\
1(4,2)\end{array}$ & 1 \\
\hline $\begin{array}{l}\text { Manipulación genitourinaria } \\
\text { no/sí }\end{array}$ & $\begin{array}{c}16(72,7 \%) \\
6(27,3 \%)\end{array}$ & $\begin{array}{c}23(95,8 \%) \\
1(4,2 \%)\end{array}$ & 0,029 \\
\hline
\end{tabular}

TABLA I (CONT.)

ANÁLISIS DESCRIPTIVO DE LAS VARIABLES ESTUDIADAS EN LOS PERIODOS 1985-86 Y 1996-97

\begin{tabular}{|c|c|c|c|}
\hline Variable & $1985-86$ & 1996-97 & $p$ \\
\hline $\begin{array}{l}\text { Manipulación vascular } \\
\text { no/sí }\end{array}$ & $\begin{array}{c}17(77,3 \%) \\
5(22,7 \%)\end{array}$ & $\begin{array}{c}23(95,8 \%) \\
1(4,2 \%)\end{array}$ & 0,09 \\
\hline $\begin{array}{l}\text { Manipulación digestiva } \\
\text { no/sí }\end{array}$ & $22(100 \%)$ & $24(100 \%)$ & 1 \\
\hline $\begin{array}{l}\text { Cirugía previa } \\
\text { no/si }\end{array}$ & $\begin{array}{l}19(86,4 \%) \\
3(13,6 \%)\end{array}$ & $\begin{array}{c}21(87,5 \%) \\
3(12,5 \%)\end{array}$ & 0,9 \\
\hline $\begin{array}{l}\text { Origen de la bacteriemia } \\
{ }^{*} \text { digestivo } \\
{ }^{*} \text { genitourinario } \\
{ }^{*} \text { desconocido } \\
{ }^{*} \text { piel } \\
{ }^{*} \text { vasculares y endocarditis } \\
{ }^{*} \text { respiratorio }\end{array}$ & $\begin{array}{c}12(54,5 \%) \\
0 \\
3(13,6 \%) \\
5(22,7 \%) \\
1(4,6 \%) \\
1(4,6 \%)\end{array}$ & $\begin{array}{c}13(54,2 \%) \\
1(4,2 \%) \\
3(12,5 \%) \\
3(12,5 \%) \\
1(4,2 \%) \\
3(12,5 \%)\end{array}$ & 0,79 \\
\hline $\begin{array}{l}\text { Hipotensión } \\
\text { no/sí }\end{array}$ & $\begin{array}{l}12(54,5 \%) \\
10(44,5 \%)\end{array}$ & $\begin{array}{c}20(83,3 \%) \\
4(16,7 \%)\end{array}$ & 0,034 \\
\hline $\begin{array}{l}\text { Trombopenia } \\
\text { no } \\
\text { sí }\end{array}$ & $\begin{array}{c}20(90,9) 20 \%) \\
2(9,1 \%)\end{array}$ & $\begin{array}{c}22(95,6 \%) \\
1(4,3 \%)\end{array}$ & 0,52 \\
\hline $\begin{array}{l}\text { Metástasis } \\
\text { no } \\
\text { sí }\end{array}$ & $\begin{array}{c}19(86,4 \%) \\
3(13,6 \%)\end{array}$ & $24(100 \%)$ & 0,32 \\
\hline $\begin{array}{l}\text { CID } \\
\text { no } \\
\text { sí }\end{array}$ & $\begin{array}{c}21(95,4 \%) \\
1(4,6 \%)\end{array}$ & $\begin{array}{c}22(95,6 \%) \\
1(4,4 \%)\end{array}$ & 0,97 \\
\hline $\begin{array}{l}\text { Tto. empírico } \\
\text { adecuado } \\
\text { inadecuado }\end{array}$ & $\begin{array}{c}17(77,3 \%) \\
5(22,7 \%)\end{array}$ & $\begin{array}{c}20(86,9 \%) \\
3(13,1 \%)\end{array}$ & 0,45 \\
\hline $\begin{array}{l}\text { Tto específico } \\
\text { adecuado } \\
\text { inadecuado }\end{array}$ & $\begin{array}{l}18(90 \%) \\
2(10 \%)\end{array}$ & $\begin{array}{c}22(95,6 \%) \\
1(4,4 \%)\end{array}$ & \\
\hline
\end{tabular}

NS: no significación estadística $(p>0,10)$

$\mathrm{n}=$ número de casos

robios, incluídas las bacteriemias polimicrobianas en la que al menos uno de los microrganismos fue anaerobio (8), (36\% de las bacteriemias por anaerobios, fueron polimicrobianas) (Tablas II y III), 10 (45\%) fueron sexo masculino y el resto femenino. Los pacientes fueron mayores de 60 años en 14 casos $(63,6 \%)$, y 6 entre 40 y 60 años $(27,27 \%)$. Los servicios hospitalarios en los que se diagnosticaron las bacteriemias por anaerobios fueron: médico, 15 (68,68\%), quirúrgico 3 (13.63\%), UCI 3(13,63\%), y hematología 1 (4,5\%). La adquisición fue extrahospitalaria en $8(36.36 \%)$ casos. Entre los factores predisponentes destacan, la cirugía previa 3 $(13,63 \%)$, tratamiento esteroideo 1 caso $(4.5 \%)$, tratamiento con otros inmunosupresores, 1 caso $(4,5 \%)$, antibioterapia previa en 7 casos $(31,82 \%)$. En cuanto al origen: 12 casos $(54,54 \%)$ fueron de origen biliar, hepatobiliar y abdominal, 
TABLA II

MICROORGANISMOS DE LAS BACTERIEMIAS POR ANAEROBIOS

\begin{tabular}{|c|c|c|c|}
\hline \multirow[b]{2}{*}{ Microorganismo } & \multicolumn{2}{|c|}{ Periodo } & \multirow[b]{2}{*}{ Total } \\
\hline & $1985-86$ & 1996-97 & \\
\hline \multirow[t]{2}{*}{ Polimicrobiana } & $17,4 \%$ & $13,0 \%$ & $30,4 \%$ \\
\hline & 13 & 14 & 27 \\
\hline \multirow[t]{2}{*}{ Bacteroides } & $28,3 \%$ & $30,4 \%$ & $58,7 \%$ \\
\hline & 1 & 1 & 2 \\
\hline \multirow[t]{2}{*}{ Fusobacterium } & $2,2 \%$ & $2,2 \%$ & $4,3 \%$ \\
\hline & & 2 & 2 \\
\hline \multirow[t]{2}{*}{ Clostridium spp } & & $4,3 \%$ & $4,3 \%$ \\
\hline & & 1 & 1 \\
\hline Flavobacterium & & $2,2 \%$ & $2,2 \%$ \\
\hline meningosepticum & 22 & 24 & 46 \\
\hline Total & $47,8 \%$ & $52,2 \%$ & 100,0 \\
\hline $\begin{array}{l}\mathrm{p}=0,51 \\
\text { 1986-87: de las } 8 \text { ba } \\
\text { gilis y } 2 \text { Clostridium } \\
\text { 1996-97: de las } 6 \text { ba } \\
\text { gilis. }\end{array}$ & s polimic & nas 6 teni & cteroi \\
\hline
\end{tabular}

TABLA III

MICROORGANISMOS DE LAS BACTERIEMIAS POLIMICROBIANAS

\begin{tabular}{|c|c|c|c|}
\hline Microorganismo & $\begin{array}{l}\text { Periodo } \\
1985-86\end{array}$ & $1996-97$ & Total \\
\hline No polimicrobiana & $\begin{array}{c}14 \\
30,4 \%\end{array}$ & $\begin{array}{c}18 \\
39,1 \%\end{array}$ & $\begin{array}{r}32 \\
69,6 \%\end{array}$ \\
\hline $\begin{array}{l}\text { Bacteroides fragilis+Streptococcus } \\
\text { faecalis }\end{array}$ & $\begin{array}{c}1 \\
2,2 \%\end{array}$ & & $\begin{array}{c}1 \\
2,2 \%\end{array}$ \\
\hline Escherichia coli+Clostridium spp & $\begin{array}{c}1 \\
2,2 \%\end{array}$ & $\begin{array}{c}1 \\
2,2 \%\end{array}$ & $\begin{array}{c}2 \\
4,3 \%\end{array}$ \\
\hline $\begin{array}{l}\text { Streptococcus microaerophilico } \\
+ \text { Bacteroides fragilis }\end{array}$ & $\begin{array}{c}1 \\
2,2 \%\end{array}$ & & $\begin{array}{c}1 \\
2,2 \%\end{array}$ \\
\hline $\begin{array}{l}\text { Clostridium perfringens+Klebsiella } \\
\text { oxytoca+Streptococcus faecalis }\end{array}$ & $\begin{array}{c}1 \\
2,2 \%\end{array}$ & & $\begin{array}{c}1 \\
2,2 \%\end{array}$ \\
\hline $\begin{array}{l}\text { Bacteroides fragilist } \\
\text { Peptostreptococcus sp }\end{array}$ & $\begin{array}{c}2 \\
4,3 \%\end{array}$ & & $\begin{array}{c}2 \\
4,3 \%\end{array}$ \\
\hline $\begin{array}{l}\text { Bacteroides fragilist } \\
\text { Escherichia coli }\end{array}$ & $\begin{array}{c}2 \\
4,3 \%\end{array}$ & & $\begin{array}{c}2 \\
4,3 \%\end{array}$ \\
\hline $\begin{array}{l}\text { Streptococcus viridanst } \\
\text { Prevotella sp }\end{array}$ & 1 & $2,2 \%$ & $\begin{array}{c}1 \\
2,2 \%\end{array}$ \\
\hline $\begin{array}{l}\text { Streptococcus sp+ } \\
\text { Fusobacterium sp }\end{array}$ & & $\begin{array}{c}1 \\
2,2 \%\end{array}$ & $\begin{array}{c}1 \\
2,2 \%\end{array}$ \\
\hline $\begin{array}{l}\text { Escherichia coli+Staphylococcus } \\
\text { epidermis+Bacteroides fragilis }\end{array}$ & & $\begin{array}{c}1 \\
2,2 \%\end{array}$ & $\begin{array}{c}1 \\
2,2 \%\end{array}$ \\
\hline $\begin{array}{l}\text { Streptococcus viridanst } \\
\text { Bacteroides fragilis }\end{array}$ & & $\begin{array}{c}1 \\
2,2 \%\end{array}$ & $\begin{array}{c}1 \\
2,2 \%\end{array}$ \\
\hline $\begin{array}{l}\text { Staphylococcus sp coagulasa } \\
\text { negativo+Bacteroides sp }\end{array}$ & & $\begin{array}{c}1 \\
2,2 \%\end{array}$ & $\begin{array}{c}1 \\
2,2 \%\end{array}$ \\
\hline Total & $\begin{array}{c}22 \\
47,8 \%\end{array}$ & $\begin{array}{c}24 \\
52,2 \%\end{array}$ & $\begin{array}{c}46 \\
100,0\end{array}$ \\
\hline
\end{tabular}

$5(22,72 \%)$ de piel, partes blandas, y herida quirúrgica, y 3 $(13,63 \%)$, de origen desconocido. En cuanto a los datos clínicos, sólo un caso presentó coagulación intravascular diseminada. En lo que se refiere al tratamiento (Tabla IV), 17 casos $(77,27 \%)$, recibieron tratamiento empírico adecuado, y 5 casos $(22,72 \%)$, inadecuado.

En cuanto al tratamiento específico 18 (81,81\%), recibieron tratamiento adecuado, y 2 casos tratamiento inadecuado. De los 18 pacientes que recibieron tratamiento específico adecuado, 16 habían recibido tratamiento empírico adecuado $(88,9 \%)$. Un paciente recibió tratamiento empírico adecuado y específico inadecuado, 1 recibió tratamiento específico y empírico inadecuado, y 2 recibieron tratamiento empírico inadecuado y específico adecuado.

En cuanto a la evolución, 12 pacientes curaron (54,54\%), y 10 pacientes fallecieron $(45,45 \%)$, de los cuales 6 habían recibido tratamiento empírico adecuado.

Finalmente los microrganismos con crecimiento fueron (Tablas II y III): 8 (36,36\%) bacteriemias polimicrobianas; 13 (59,09\%) Bacteroides sp; 1 (4,54\%) Fusobacterium.

\section{AÑ $1996-97$}

Se obtuvieron un total 4.958 hemocultivos, de los que fueron positivos $1.100(22,1 \%)$, y verdaderos fueron 472 $(42,9 \%)$. De estos, $24(5,08 \%)$ fueron bacteriemias por anaerobios, incluídas las bacteriemias polimicrobianas en la que al menos uno de los microrganismos fue anaerobio $(6,25 \%$ de las bacteriemias por anaerobios, fueron polimicrobianas). 13 $(54,16 \%)$ fueron sexo masculino y el resto femenino. Los pacientes fueron mayores de 60 años en 18 casos (75\%).Los servicios hospitalarios en los que se diagnosticaron las bacteriemias por anaerobios fueron: médico, 13 (54,16\%), quirúrgico $9(37,5 \%)$, UCI 2 (8,33\%). La adquisición fue extrahospitalaria en $15(62,5 \%)$ casos. Entre los factores predisponentes destacan, la cirugía previa $3(12,5 \%)$, tratamiento esteroideo 1 caso $(4,16 \%)$, tratamiento con otros inmunosupresores, 1 caso $(4,16 \%)$, antibioterapia previa en 6 casos $(25 \%)$. En cuanto a las manipulaciones 1 caso $(4,16 \%)$ recibió manipulación genitourinaria; 1 caso $(4,16 \%)$, manipulación respiratoria, y 1 caso $(4,16 \%)$ vascular. En cuanto al origen: 13 casos $(54,16 \%)$ fueron de origen biliar, hepatobiliar y abdominal; 3 (12,5\%) de piel, partes blandas, y herida quirúrgica, y 3 (12,5\%), de origen desconocido. En cuanto a los datos clínicos, sólo un caso presentó coagulación intravascular diseminada. En lo que se refiere al tratamiento (Tabla IV), 20 casos $(83,33 \%)$, recibieron tratamiento empírico adecuado, y 3 casos $(12,5 \%)$, inadecuado. En cuanto al tratamiento específico $21(91,66 \%)$, recibieron tratamiento adecuado, y 1 caso tratamiento inadecuado. De los 21 pacientes que recibieron tratamiento específico adecuado, 20 habían recibido tratamiento empírico adecuado $(95,2 \%)$. En cuanto a la evolución, 14 pacientes curaron $(63,6 \%)$, y 8 pacientes fallecieron $(36,6 \%)$, de los cuales todos habían recibido tratamiento empírico adecuado. Hubo un paciente que falleció y que recibió tratamiento empírico inadecuado, sin tiempo a recibir tratamiento específico adecuado. Finalmente los microrganismos se exponen en (Tablas II y III): $6(25 \%)$ bacteriemias polimicrobianas; $14(58,33 \%)$ Bacteroides sp; 1 (4,16\%) Fusobacterium; 2 (8,33\%) Clostridium sp; 1 (4,16\%) Flavobacterium meningosepticum. 


\begin{tabular}{|c|c|c|c|c|c|c|}
\hline \multirow{2}{*}{ Tratamiento empírico } & \multirow[b]{2}{*}{ Periodo } & & & \multicolumn{2}{|c|}{ Evolución } & \multirow[b]{2}{*}{ Total } \\
\hline & & & & Curación & Muerte & \\
\hline \multirow[t]{4}{*}{ Adecuado } & $1985-86$ & Tratamiento específico & $\begin{array}{l}\text { Adecuado } \\
\text { Inadecuado }\end{array}$ & $\begin{array}{c}10 \\
58,8 \% \\
1 \\
5,9 \%\end{array}$ & $\begin{array}{c}6 \\
35,3 \%\end{array}$ & $\begin{array}{c}16 \\
94,1 \% \\
1 \\
5,9 \%\end{array}$ \\
\hline & & Total & & $\begin{array}{c}11 \\
64,7 \%\end{array}$ & $\begin{array}{c}6 \\
35,3 \%\end{array}$ & $\begin{array}{c}17 \\
100,0 \%\end{array}$ \\
\hline & $1996-97$ & Tratamiento específico & Adecuado & $\begin{array}{c}12 \\
60,0 \%\end{array}$ & $\begin{array}{c}8 \\
40,0 \%\end{array}$ & $\begin{array}{c}20 \\
100,0 \%\end{array}$ \\
\hline & & Total & & $\begin{array}{c}12 \\
60,0 \%\end{array}$ & $\begin{array}{c}8 \\
40,0 \%\end{array}$ & $\begin{array}{c}20 \\
100,0 \%\end{array}$ \\
\hline \multirow[t]{4}{*}{ Inadecuado } & $1985-86$ & Tratamiento específico & $\begin{array}{l}\text { Adecuado } \\
\text { Inadecuado }\end{array}$ & $\begin{array}{c}1 \\
33,3 \%\end{array}$ & $\begin{array}{c}2 \\
66,7 \%\end{array}$ & $\begin{array}{c}2 \\
66,7 \% \\
1 \\
33,3 \%\end{array}$ \\
\hline & & Total & & $\begin{array}{c}1 \\
33,3 \%\end{array}$ & $\begin{array}{c}2 \\
66,7 \%\end{array}$ & $\begin{array}{c}3 \\
100,0 \%\end{array}$ \\
\hline & $1996-97$ & Tratamiento específico & $\begin{array}{l}\text { Adecuado } \\
\text { Inadecuado }\end{array}$ & $\begin{array}{c}1 \\
50,0 \% \\
1 \\
50,0 \%\end{array}$ & & $\begin{array}{c}1 \\
50,0 \% \\
1 \\
50,0 \%\end{array}$ \\
\hline & & Total & & $\begin{array}{c}2 \\
100,0 \%\end{array}$ & & $\begin{array}{c}2 \\
100,0 \%\end{array}$ \\
\hline
\end{tabular}

Las variables con diferencias estadísticamente significativas con $\mathrm{p}<0,10$ entre el periodo $1985-86$ y 1996-97 fueron: adquisición más frecuente intrahospitalaria (p 0,07); manipulaciones genitourinarias ( $\mathrm{p}$ de 0,02 ); manipulaciones vasculares ( $\mathrm{p}$ de 0,06$)$ y la presencia de hipotensión ( $\mathrm{p}$ de 0,034$)$ que fueron más frecuentes durante el primer periodo que durante el segundo. Sin embargo al analizar la evolución a curación o muerte durante el primer periodo y durante el segundo, se observa que no existe diferencia estadísticamente significativa aunque el porcentaje de curaciones fue mayor durante el segundo periodo (15 casos $62,5 \%$ ) que durante el primero (12 casos $54,6 \%)$.

\section{DISCUSIÓN}

En primer lugar mencionar que la incidencia porcentual de las bacteriemias por anaerobios respecto al global de las bacteriemias es baja. A diferencia de los datos presentados por otros autores (11), esta ha aumentado discretamente en 10 años (aunque la diferencia no es estadísticamente significativa probablemente por el bajo número de casos). Es importante mencionar que la incidencia se refiere al porcentaje del número de bacteriemias por anaerobios respecto al número total de bacteriemias verdaderas diagnosticadas, bacteriemias producidas tanto en pacientes ingresados como en pacientes no ingresados; y probablemente dar el valor de la incidencia respecto a bacteriemias verdaderas sea más adecuado que darlo en relación al número de ingresos en cada periodo(ya que las bacteriemias se han recogido tanto en pacientes ingresados como en no ingresados),o que darlo en relación al número total de hemocultivos realizados en cada periodo (puesto que no están protocolizados los criterios de extracción de hemocultivos).

Por otro lado, el cambio en el modo de procesamiento de los hemocultivos (método manual vs. método automatizado) en ambos periodos, podría limitar los resultados obtenidos; no obstante las opciones diagnósticas de bacteriemia son las que existen en cada periodo, y son similares en dicho periodo tanto en las bacteriemias por aerobios como por anaerobios, no pudiendo valorarse de otra forma.

Las características de las bacteriemias por anaerobios tanto clínicas (foco de origen más frecuente el abdominal, seguido de piel, partes blandas, y herida quirúrgica), y más frecuentes en pacientes por encima de los 60 años se mantienen constantes en ambos periodos, así como que Bacteroides fragilis es mayoritariamente el microrganismo más frecuentemente aislado en estas bacteriemias, y esto coincide con lo ya publicado por otros autores $(4,10)$. 
Un dato interesante es el gran porcentaje de tratamientos empíricos adecuados, que se ha mantenido en ambos periodos, hecho que haría plantearse la posibilidad de suprimir los medios de cultivo para anaerobios, como algunos autores propugnan, basándose en el hecho de que las bacteriemias por anaerobios serían predecibles $(13,14)$. Este dato vendría apoyado además por el hecho observado durante el segundo periodo en el que los 8 pacientes que fallecieron del total de 9 , habían recibido tratamiento empírico adecuado (Tabla IV). Bien es verdad que frente a esta afirmación se podría mencionar que el número de casos es bajo, o que los tratamientos empíricos son correctos porque el arsenal antibiótico en general es de muy amplio espectro, y ello podría implicar la realización de tratamientos empíricos adecuados pese a no predecir clínicamente a priori una posible bacteriemia por anaerobios. Esta afirmación no coincide sin embargo con lo expuesto por otros estudios en los que el tratamiento empírico es modificado en un porcentaje importante de casos, hasta en el $50 \%$ en algunos estudios (15), hecho que influye además en la mortalidad por bacteriemia por anaerobios según dichos estudios (3).

Una segunda pregunta a realizarse es si el conocimiento o no de los microrganismos causantes de bacteriemia por anaerobios, supone un cambio en el manejo de pruebas diagnósticas, y que ello derive además en un cambio pronóstico en el paciente no a corto plazo, sino a medio plazo, como pudiera ser la búsqueda de causas que pudieran favorecer una bacteriemia por ejemplo por Prevotella sp o Fusobacterium sp, en pacientes con enfermedad periodontal y que pueden ser causa de infecciones pleuropulmonares futuras por ejemplo, o la adopción de medidas más agresivas a nivel diagnóstico en el caso de bacteriemias por Bacteroides $s p$.

Quizás una alternativa a lo ya mencionado sería tratar de establecer un modelo predictivo de bacteriemia por anaerobios, y realizar la extracción de hemocultivos en dicho medio de cultivo en caso de sospecha clínica tras la aplicación de dicho modelo. La réplica no obstante a esta posibilidad es saber que en los medios de cultivo para anaerobios no sólo crecen microrganismos anaerobios, sino aerobios-anaerobios facultativos $(16,17)$ como pueden ser las enterobacterias, y probablemente la supresión de dichos medios de crecimiento para anaerobios conlleven una disminución general de la rentabilidad de los hemocultivos. Una solución final sería tratar de establecer un modelo predictivo de bacteriemia en general (18), aumentando el volumen de extracción en medios de cultivos sólo para aerobios, y un modelo particular predictivo de bacteriemia por anaerobios, realizando extracción y medio de cultivo para anaerobios sólo en el caso en el que el modelo predictivo así lo sugiera, tratando así de mantener la rentabilidad diagnóstica de la prueba "hemocultivo", y probablemente con disminución de costos en relación a la realización de las mismas al aplicar a todo paciente el modelo predictivo para realizar o no hemocultivos y dentro de ellos extracción o no en medio para anaerobios.

En relación a las otras variables con diferencias estadísticamente significativas en ambos periodos, y concretamente las manipulaciones genitourinarias, y las manipulaciones vasculares, su influencia real sobre las bacteriemias por anaerobios debe ser mínima puesto que analizados los orígenes de las bacteriemias, éstos son excepcionales (tanto el foco urinario como el vascular) (Tabla I). En cuanto a la variable hipotensión, que como característica de los pacientes es significativamente superior en el primer periodo que en el segundo, influye también de forma significativa en la mortalidad de las bacteriemias del periodo $1985-86 \mathrm{p}=0,035$, pero no en la mortalidad del segundo periodo p 0,57 , simplemente por el mero de hecho del bajo número de pacientes hipotensos en el momento de la bacteriemia (Tabla I) hecho que podría justificar al menos en parte la mayor mortalidad del primer periodo $(45,4 \%)$ en relación al segundo $(37,5 \%)$ aunque la diferencia no es estadísticamente significativa. Dicho dato coincide con el ya publicado por los autores en relación con las bacteriemias en general y los factores pronósticos de mortalidad, en los que la presencia de hipotensión se asocia de forma independiente con la evolución a muerte de las bacteriemias (19).

Así pues pese a la baja incidencia de bacteriemias por anaerobios y el alto porcentaje de tratamientos empíricos adecuados, la recomendación de los autores es mantener los medios de cultivo para anaerobios, porque probablementede de forma global aumenta la rentabilidad de los hemocultivos por crecimiento de microrganismos aerobios-anaerobios facultativos, en espera de la realización de nuevos estudios que confirmen el mantenimiento de la rentabilidad global de los hemocultivos aumentando el volumen de extracción en medio de crecimiento sólo para aerobios, así como de estudios que demuestren la utilidad o no del diagnóstico de bacteriemias por anaerobios no sólo a corto plazo sino a medio plazo como ya se ha comentado, con la posible utilización de un modelo predictivo para las mismas.

\section{AGRADECIMIENTOS}

A la Dra. Del Rey y al Dr. López Brea, del Servicio de Microbiología, por su colaboración.

\section{Bibliografía}

1. Lombardi DP, Engleberg C. Anaerobic bacteremia: Incidence, patient characteristics, and clinical significance. Am J Med 1992; 92: 53-60.

2. Arpi M, Renneberg J, Andersen HK, Nielsen B, Larsen SO. Bacteremia at a Danish university hospital during a twenty five-year period (19681992). Scand J Infect Dis 1995; 27: 245-51.

3. Salonen JH, Eerola E, Meurman O. Clinical significance and outcome of anaerobic bacteremia. Clin Infect Dis 1998; 26: 1413-7.

4. Gómez J, Banos V, Ruiz J, Herrero F, Pérez M, Pretel L, et al. Clinical significance of anaerobic bacteremias in a general hospital. A prospecti- ve study from 1998 to 1992 . Clin Investig 1993; 71: 595-9.

5. Bouza E, García de la Torre M. Bacteriemia y endocarditis por anaerobios. En: García Rodríguez JA, ed. Infecciones por anaerobios. Barcelona. JR Prous, 1991; 39-51.

6. Dorsher CW, Wilson WR, Rosenblatt JE. Anaerobic bacteremia and cardiovascularinfections. En Finegold SM, George WL, eds. Anaerobic infections in humans. San Diego: Academic Press 1989; 289-310.

7. Zaidi AK, Knaut AL, Misrett S, Reller LB. Value of routine anaerobic blood cultures for pediatric patients. J Pediatr 1995; 127: 263-8. 
8. Kornfski R, Schwartz D, Averluch M, Levo Y, Berger S, Giladi M. Anaerobic bacteremia: a retrospective four year analysis in general medicine and cancer patients. Infection 1993; 21: 241-4

9. Spanik S, Trupl J, Kunova A, Pichna P, Helpianska L, Ilavska I, et al. Bloodstream infections due to anaerobic bacteria in cancer patients: epidemiology, etiology, risk factors, clinical presentation and outcome of anaerobic bacteremia. Neoplasma 1996; 43: 235-8.

10. Goldstein EJ. Anaerobic bacteremia Clin Infect Dis 1996; 23 (Supl. 1): S97-101.

11. Dorsher CW, Rosenthablatt JE, Wilson WR and Ilsprup DM. Anaerobic Bacteriemia: Decreasing Rate Over a 15 Year Period. Rev Infect Dis 1991; 13: 633-6.

12. Murray PR, Traynor P, Hopson D. Critical assessment of blood culture techniques: analysis of recovery of obligate and facultative anaerobes, strict aerobic bacteria and fungi in aerobic and anaerobic blood culture bottles. J Clin Microbiol 1992; 30: 1462-8.

13. Ortiz E, Sande MA .Routine use of anaerobic blood cultures: are they still indicated? Am J Med 2000; 108: 505-6.

14. Pottumarthy S, Morris AJ. Assessment of the yield of anaerobic blood cultures. Pathology 1997; 29: 415-7.

15. Peraino VA, Cross SA, Goldstein EJ. Incidence and clinical significance of anaerobic bacteriemia in a community hospital. Clin Infect Dis 1993; 16 (Supl. 4): S 288-91.

16. Cockerill FR, Hughes JG, Vetter EA, Mueller RA, Weaver AL, Ilstrup DM, et al. Analysis of 281,797 consecutive blood cultures performed over an eight-year period: trends in microorganisms isolated and the value of anaerobic culture of blood. Clin Infect Dis 1997; 24: 403-18 .

17. Ruiz Giardin JM, Del Rey MC, Serrano M, Garcia G, González P, Isasia T. Utilidad de los medios de crecimiento para anaerobios en las bacteriemias de origen extrahospitalario. An Med Interna (Madrid) 2003 20: $179-182$.

18. Bates D, Sands K, Miller E, Lanken P, Hibberd P, Graman P, et al. Predicting Bacteremia in Patients with Sepsis Syndrome. J Infect Dis 1997; 176: 1538-51.

19. Noguerado A., Ruiz JM, Pizarro A, Méndez J, La Hulla F, Fernández M et al .Análisis de factores pronósticos de mortalidad de las bacteriemias y fungemias en un Hospital Universitario. Evolución en 10 años. Rev Clin Esp 2001; 201: 122-129. 